\title{
OCT-measured plaque free wall angle is indicative for plaque burden: overcoming the main limitation of OCT?
}

\author{
Ayla Hoogendoorn ${ }^{1}\left(\right.$ ID $\cdot$ Muthukaruppan Gnanadesigan $^{1} \cdot$ Guillaume Zahnd $^{2}$. \\ Nienke S. van Ditzhuijzen ${ }^{3} \cdot$ Johan C. H. Schuurbiers $^{1} \cdot$ Gijs van Soest $^{1}$. \\ Evelyn $\operatorname{Regar}^{3} \cdot$ Jolanda J. Wentzel ${ }^{1}$
}

Received: 26 May 2016 / Accepted: 13 July 2016 / Published online: 20 July 2016

(C) The Author(s) 2016. This article is published with open access at Springerlink.com

\begin{abstract}
The aim of this study was to investigate the relationship between the plaque free wall (PFW) measured by optical coherence tomography (OCT) and the plaque burden (PB) measured by intravascular ultrasound (IVUS). We hypothesize that measurement of the PFW could help to estimate the PB, thereby overcoming the limited ability of OCT to visualize the external elastic membrane in the presence of plaque. This could enable selection of the optimal stent-landing zone by OCT, which is traditionally defined by IVUS as a region with a $\mathrm{PB}<40 \%$. PB (IVUS) and PFW angle (OCT and IVUS) were measured in 18 matched IVUS and OCT pullbacks acquired in the same coronary artery. We determined the relationship between OCT measured PFW (PFW ${ }_{\mathrm{OCT}}$ ) and IVUS PB ( $\left.\mathrm{PB}_{\mathrm{IVUS}}\right)$ by non-linear regression analysis. An ROC-curve analysis was used to determine the optimal cut-off value of PFW angle for the detection of $\mathrm{PB}<40 \%$. Sensitivity, specificity, positive predictive value (PPV) and negative predictive value (NPV) were calculated. There is a significant correlation between $\mathrm{PFW}_{\mathrm{OCT}}$ and $\mathrm{PB}_{\text {IVUS }}\left(\mathrm{r}^{2}=0.59\right)$. The
\end{abstract}

Evelyn Regar and Jolanda J. Wentzel shared last authorship.

$\triangle$ Ayla Hoogendoorn

a.hoogendoorn@erasmusmc.nl

1 Biomechanics Laboratory, Department of Cardiology, Biomedical Engineering, EE2334b, Erasmus MC, PO Box 2040, 3000 CA Rotterdam, The Netherlands

2 Biomedical Imaging Group Rotterdam, Departments of Radiology \& Nuclear Medicine and Medical Informatics, Erasmus MC, Rotterdam, The Netherlands

3 Interventional Cardiology, Department of Cardiology, Erasmus MC, Rotterdam, The Netherlands optimal cut-off value of the $\mathrm{PFW}_{\mathrm{OCT}}$ for the prediction of a $\mathrm{PB}_{\text {IVUS }}<40 \%$ is $\geq 220^{\circ}$ with a PPV of $78 \%$ and an NPV of $84 \%$. This study shows that $\mathrm{PFW}_{\mathrm{OCT}}$ can be considered as a surrogate marker for $\mathrm{PB}_{\text {IVUS }}$, which is currently a common criterion to select an optimal stentlanding zone.

Keywords Optical coherence tomography Intravascular ultrasound - Plaque free wall · Plaque burden $\cdot$ Stent-landing zone

\section{Introduction}

In recent years, intravascular optical coherence tomography (OCT) has emerged as imaging technique for guiding percutaneous coronary intervention (PCI). OCT offers a very high spatial and lateral resolution compared to intravascular ultrasound (IVUS). However, this comes at the expense of penetration depth into the tissue [1]. Based on IVUS studies $[2,3]$, the plaque burden $(\mathrm{PB})$ is a leading criterion for a stent-landing zone, where areas with $\mathrm{PB}<40 \%$ [4] are considered optimal. The ability of OCT to visualize PB is limited by optical attenuation: the signal decreases with depth in tissue. Either a thick layer of fibrous (low-attenuation) plaque or the presence of superficial lipids or dense macrophage accumulations (high-attenuation) [5], may obscure the visibility of the media behind the plaque. This potentially limits the use of OCT to guide stent implantation when it comes to the selection of the optimal stent landing zones within the target artery. More recently, in addition to low plaque burden, the absence of lipid/necrotic core within the landing zone has been advocated as a decision criterion which can very reliably be identified by OCT, in contrast to IVUS [6]. 
In a previous study [7], we demonstrated a strong inverse linear relationship between $\mathrm{PB}$ and the plaque free wall (PFW) angle using IVUS imaging ( $\mathrm{PB}_{\text {IVUS }}$ and $\left.\mathrm{PFW}_{\text {IVUS }}\right)$. In the present study we hypothesize that the angle of the PFW measured in OCT $\left(\mathrm{PFW}_{\mathrm{OCT}}\right.$ ) can likewise reflect the $\mathrm{PB}$. Second, we postulate that regions with a $\mathrm{PB}<40 \%$ can be selected for finding an optimal stent-landing zone. When the PFW angle is small $\left(<180^{\circ}\right)$, it might be instantly clear that disease is present with a $\mathrm{PB}>40 \%$, thus forming a suboptimal stent-landing zone. Similarly, sections with a large PFW angle $>270^{\circ}$ can be easily and reliably identified by OCT as optimal stent-landing zones with a $\mathrm{PB}<40 \%$. However, in the intermediate region between $180^{\circ}-270^{\circ} \mathrm{PFW}$, the interpretation might be more ambiguous. The aim of this study was to investigate the relationship between $\mathrm{PFW}_{\mathrm{OCT}}$ and $\mathrm{PB}_{\mathrm{IVUS}}$ and establish the utility (expressed in predictive values for $\mathrm{PB}<40 \%$ ) of this relationship to detect $\mathrm{PB}<40 \%$ for selection of the optimal stent landing zone by OCT.

\section{Materials and methods}

\section{Study population}

To study the predictive value of the $\mathrm{PFW}_{\mathrm{OCT}}$ for determination of the $\mathrm{PB}$, we performed a retrospective observational study. NIRS-IVUS (TVC, InfraRedx, Burlington, Massachusetts, USA) and OCT (St. Jude Medical Inc., St. Paul, MN, USA) pullbacks of the same ROI were used from 18 left anterior descending (LAD) coronary arteries of 18 patients with stable or unstable coronary syndrome included in the OC3T study (Erasmus MC, Rotterdam).

The NIRS-IVUS images were acquired using a commercially available hybrid optical/ultrasound catheter with an automated pullback (pullback speed $0.5 \mathrm{~mm} / \mathrm{s}$ and 16 frames per second). The OCT images were obtained with C7-XR/ Illumien and Dragonfly catheter. Automated OCT pullback (pullback speed $20 \mathrm{~mm} / \mathrm{s}$ ) was performed during simultaneous iso-osmolar X-ray contrast medium (Visipaque 320, GE Healthcare, Buckinghamshire, U.K.) delivery through the guide catheter, using a power injector (Medrad ProVis, Bayer HealthCare LLC, NJ, USA; typical flush rate $3.0 \mathrm{ml} / \mathrm{s}$ ).

\section{Analysis}

The OCT and IVUS pullbacks were matched by overlaying the frames with the same side branches. The remainder of the frames was linearly interpolated in between the side branches. Matched NIRS-IVUS and OCT images were analyzed every millimeter over a ROI of the most proximal $20 \mathrm{~mm}$ of a pullback. On the IVUS images, the external elastic lamina (vessel area $=$ VA) and lumen area (LA) contours were drawn using QCU-CMS software (version 4.69, Leiden University Medical Centre, LKEB, Division of Image Processing). These contours were used for the calculation of the plaque area $(\mathrm{PA}=\mathrm{VA}-\mathrm{LA})$ and subsequently the $\mathrm{PB}(\mathrm{PA} / \mathrm{VA} \times 100 \%)$. PFW angle was defined from the center of the lumen as the arc of the cross-section with a visible, healthy wall having an intima-media thickness of less than $0.5 \mathrm{~mm}$ in both imaging modalities. In total, 360 frames were analyzed on both the IVUS and OCT images. The frame data were averaged over $3 \mathrm{~mm}$ sections to increase robustness and to compensate for imprecise matching. Images that showed a side branch either on IVUS or OCT, with the wall out of view or a bad flush were excluded. This resulted in 106 matched IVUS and OCT $3 \mathrm{~mm}$ sections suitable for analysis. A total of 10 out of the 18 pullbacks of OCT and IVUS were analyzed by two independent expert observers for assessment of the reproducibility.

\section{Statistics}

Statistical analysis was performed using SPSS software (version 21.0, SPSS Inc., Chicago, IL, USA). Findings were regarded significant when $p<0.05$. The reproducibility of all PFW and PB measurements was assessed by performing an inter-observer agreement analysis to calculate the intraclass correlation coefficient.

Both a linear and a non-linear model were used to assess the relation between $\mathrm{PFW}_{\text {IVUS/OCT }}$ and $\mathrm{PB}_{\text {IVUS }}$. The nonlinear model was defined as follows:

$$
\begin{aligned}
& P B_{I V U S}=\mathrm{a} \times P F W+\mathrm{b} \text { for } P F W \leq \frac{(\mathrm{e}-\mathrm{b})}{(\mathrm{a}-\mathrm{d})} ; \\
& P B_{I V U S}=\mathrm{d} \times P F W+\text { e for } P F W>\frac{(\mathrm{e}-\mathrm{b})}{(\mathrm{a}-\mathrm{d})} ;
\end{aligned}
$$

and was computed for both $\mathrm{PFW}_{\mathrm{OCT}}$ and $\mathrm{PFW}_{\text {IVUS. For }}$ the non-linear regression analysis, the following starting parameters were chosen: $a=0 ; b=55 ; d=-0.2 ; e=100$. We denote the crossover value between both linear regimes as $\mathrm{PFW}_{\mathrm{C}}=(\mathrm{e}-\mathrm{b}) /(\mathrm{a}-\mathrm{d})$, resulting from the regression.

To test whether the slope of the relationship between $\mathrm{PFW}_{\text {OCT }}$ vs $\mathrm{PB}_{\text {IVUS }}$ and $\mathrm{PFW}_{\text {IVUs }}$ vs $\mathrm{PB}_{\text {IVUs }}$ was similar, implying that with $\mathrm{PFW}_{\mathrm{OCT}}$ and $\mathrm{PFW}_{\text {IVUS }}$ the same $\mathrm{PB}$ is predicted, a student's $t$ test for two independent samples was used. An ROC-curve analysis was performed to find the optimal cut-off value of $\mathrm{PFW}_{\mathrm{OCT}}$ to predict a $\mathrm{PB}_{\text {IVUS }}<40 \%$. Based on the optimal cut-off value, the sensitivity, specificity, positive predictive value (PPV) and negative predictive value $(\mathrm{NPV})$ of $\mathrm{PFW}_{\mathrm{OCT}}$ for prediction of $\mathrm{PB}_{\mathrm{IVUS}}<40 \%$ were calculated. 


\section{Results}

\section{Relation between PFW and PB in IVUS}

$\mathrm{PFW}_{\text {IVUS }}$ and $\mathrm{PB}_{\mathrm{IVUS}}$ were significantly, inversely related $\left(r^{2}=0.78\right)$ which confirmed the findings of our previous publication [7]. However, a closer fit to the model was found by applying a non-linear regression line $\left(\mathrm{r}^{2}=0.82\right)$ (Fig. 1a), with $\mathrm{PFW}_{\mathrm{C}}=182^{\circ}$.

\section{Predictive value of $P F W_{O C T}$}

To assess whether the $\mathrm{PFW}_{\mathrm{OCT}}$ could serve as a surrogate marker for $\mathrm{PB}_{\text {IVUS }}$, the $\mathrm{PFW}_{\mathrm{OCT}}$ values were plotted against the $\mathrm{PB}_{\mathrm{IVUS}}$ values. Again, a non-linear regression relationship proved significant $\left(\mathrm{r}^{2}=0.59\right)$ (Fig. 1b) with $\mathrm{PFW}_{\mathrm{C}}=186^{\circ}$. Interestingly, both IVUS and OCT show the same slope (student's $t$ test for inequality of slopes; $\mathrm{p}=\mathrm{NS}$ ) to predict $\mathrm{PB}$ for $\mathrm{PFW} \geq 186^{\circ}$. For $\mathrm{PFW}<186^{\circ}, \mathrm{PFW}_{\mathrm{OCT}}$ had no clear relationship with the $\mathrm{PB}$, contrasting the $\mathrm{PFW}_{\text {IVUS }}$ which still showed a weak relationship with the PB (Fig. 1c).

To find the optimal cut-off value of the $\mathrm{PFW}_{\mathrm{OCT}}$ to predict a $\mathrm{PB}_{\mathrm{IVUS}}<40 \%$, an ROC-curve analysis was performed. Optimization of sensitivity, specificity, PPV and NPV resulted in a $\mathrm{PFW}_{\mathrm{OCT}}$ cut-off value of $\geq 220^{\circ}$ (Fig. 2). With this cut-off value, an optimal stent-landing zone ( $\mathrm{PB}<40 \%)$ could be predicted correctly in $78 \%$ of the cases (PPV) (Fig. 3). Just as important, the NPV was $84 \%$, meaning that if the PFW angle is $<220^{\circ}, \mathrm{PB}>40 \%$ and thus forming a suboptimal stent-landing zone in $84 \%$ of the cases. This high negative predictive value will largely prevent stenting too far into a health vessel region $(\mathrm{PB}<40 \%)$.

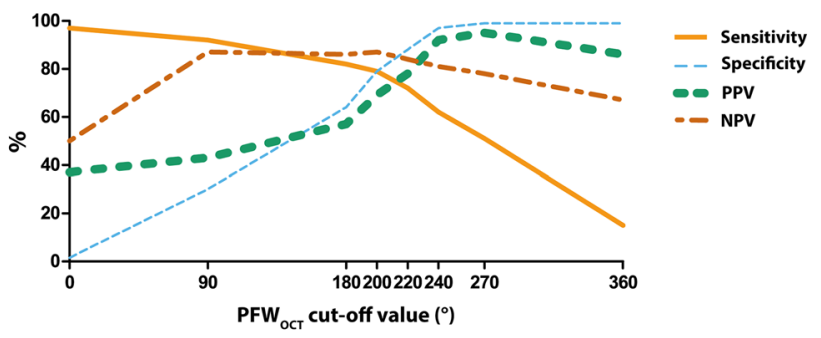

Fig. 2 Sensitivity, specificity, PPV and NPV values for different $\mathrm{PFW}_{\mathrm{OCT}}$ angle cut-off points to predict a $\mathrm{PB}<40 \%$. The optimal cutoff point is set at $220^{\circ} \mathrm{PFW}_{\mathrm{OCT}}$

As an angle of 220 degrees might be difficult to judge by simple eyeballing in the cathlab, we investigated the predictive value of a PFW $>180$ degrees for $\mathrm{PB}<40 \%$. Despite the strong relationship between $\mathrm{PFW}_{\mathrm{OCT}}$ and $\mathrm{PB}_{\mathrm{IVUS}}$, regions with a PFW angle $>180^{\circ}$ can be regarded as an optimal stent landing zone with a $\mathrm{PB}<40 \%$ in only $57 \%$ of the cases. So strikingly, in $43 \%$ of the cases the disease is more severe with a $\mathrm{PB}>40 \%$, even with only half of the circumference being occupied by plaque.

\section{Reproducibility}

Inter-observer agreement analyses of the PFW measurements were performed in 189 OCT and 177 IVUS frames and showed high intraclass correlation coefficients of respectively $0.956(95 \%$ CI $0.941-0.967)$ and $0.912(95 \%$ CI $0.884-0.934)$. The intraclass correlation coefficient of the $\mathrm{PB}_{\text {IVUS }}$ measurements was equally high at $0.886(95 \%$ CI 0.847-0.915).
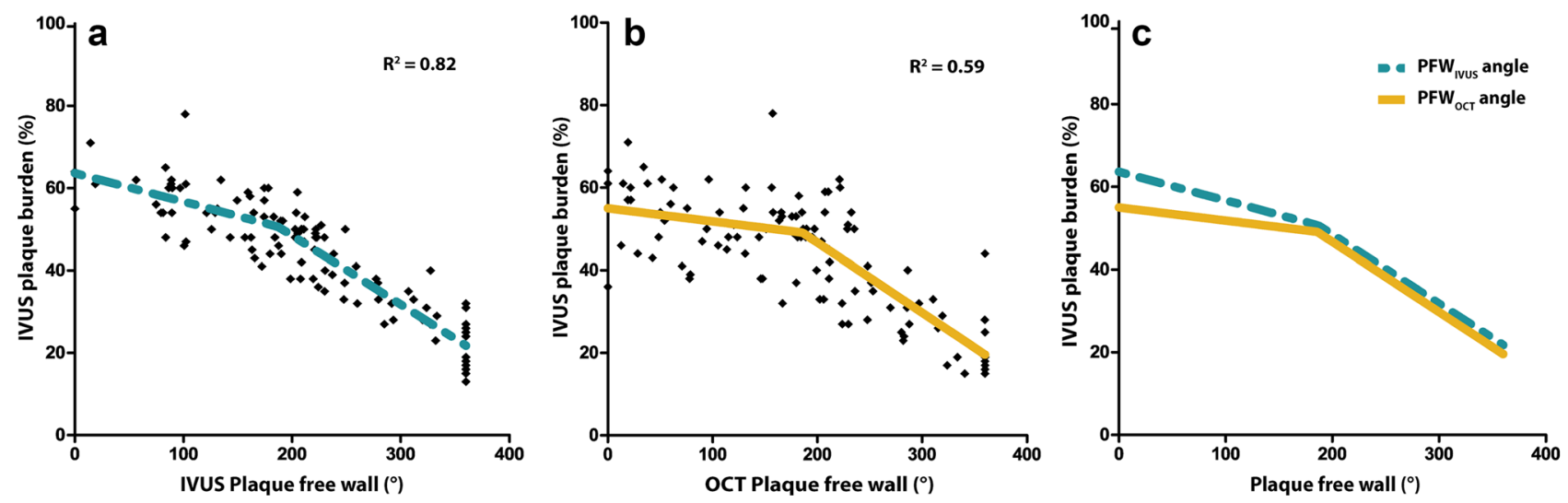

Fig. 1 Relationship between the plaque free wall (PFW) angle and plaque burden (PB). a The $\mathrm{PFW}_{\text {IVUS }}$ shows a strong inverse, non-linear correlation with the $\mathrm{PB}_{\text {IVUS }}$ with a crossover point $\mathrm{PFW}_{\mathrm{C}}=182^{\circ} \mathrm{PFW}$. Final parameters: $a=-0.069 ; b=63.59 ; d=-0.163 ; e=80.74$. b A similar relationship is seen for the $\mathrm{PFW}_{\mathrm{OCT}}$ angle with the $\mathrm{PB}_{\text {IVUS }}$ with a crossover point $\mathrm{PFW}_{\mathrm{C}}=186^{\circ}$. Final parameters: $\mathrm{a}=-0.032 ; \mathrm{b}=54.98$; $\mathrm{d}=-0.169 ; \mathrm{e}=80.59$. $\mathbf{c}$ Overlay of regression lines of (a) and (b). $\mathrm{PFW}_{\mathrm{OCT}}$ and $\mathrm{PFW}_{\text {IVUS }}$ perform equally well in predicting $\mathrm{PB}$ in the region of $\mathrm{PFW}$ angle $>186^{\circ}$. In the more diseased regions, the predictive value of the $\mathrm{PFW}_{\mathrm{OCT}}$ angle is reduced compared to $\mathrm{PFW}_{\text {IVUS }}$ 


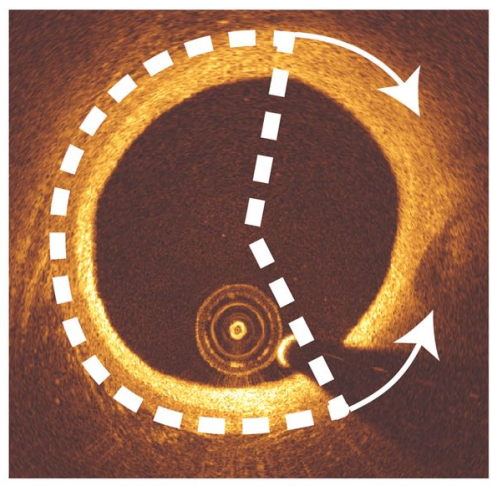

$\geq 220^{\circ}$
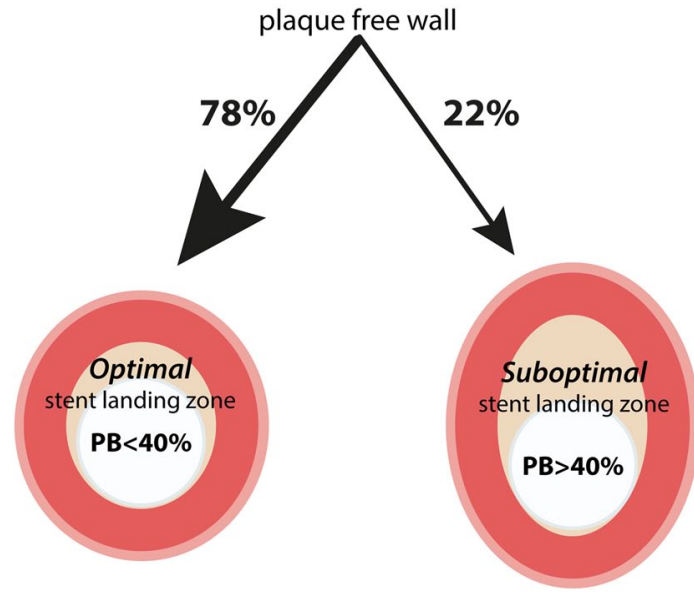

Fig. 3 Clinical application of $\mathrm{PFW}_{\mathrm{OCT}}$ angle detection. When a $\mathrm{PFW}_{\mathrm{OCT}}$ of $\geq 220^{\circ}$ is detected, in $78 \%$ of the cases this indicates the presence of a $\mathrm{PB}<40 \%$, forming an optimal stent landing zone

\section{Discussion}

This study shows for the first time that $\mathrm{PFW}_{\mathrm{OCT}}$ can be considered as a surrogate marker for $\mathrm{PB}$, which is currently a common criterion to determine stent-landing zone. In regions with a PFW angle $>186^{\circ}$, the $\mathrm{PFW}_{\mathrm{OCT}}$ has the same predictive value compared to $\mathrm{PFW}_{\text {IVUS }}$. The optimized cutoff value to predict optimal stent landing zone $(\mathrm{PB}<40 \%)$ is $\geq 220^{\circ} \mathrm{PFW}_{\text {OCT }}$.

There is a precarious balance between adequate lesion coverage and using too long stents. When the edge of the stent lands in a plaque area, it poses a risk on plaque disruption and edge dissections on a short-term and in-stent restenosis on a long-term [8]. On the other hand, placing longer stents might prove technically more challenging and might increase the risk on in-stent restenosis and stent thrombosis, even in drug eluting stents [9-11]. For this reason, determining an optimal stent-landing zone is critical for the prevention of future stent-related adverse events.

An optimal stent-landing zone is a region without lipids and a $\mathrm{PB}<40 \%$. In contrast to IVUS, OCT is capable of detecting lipids, but the limited penetration depth hampers PB measurements. OCT can however reliably detect the healthy vessel wall. Therefore, we aimed to investigate if the presence of a normal wall is helpful to predict PB by OCT. This study showed that a measurement of the PFW angle provides a reliable estimate of the $\mathrm{PB}$, without the need to fully visualize the outer wall of the vessel. Together with the fact that OCT is one of the most reliable techniques to structurally assess the presence of a lipid rich plaque, determining the stent-landing zone by OCT becomes even more feasible $[12,13]$.

The detection of the PFW in OCT can be influenced by the composition of the intima. Plaque components like a lipid/necrotic core and sites of inflammatory activity cause high attenuation and may obscure the vessel wall layers, even in a relatively thin intima. Plaque-free regions (IMT $<0.5 \mathrm{~mm}$ ) with a superficial layer of macrophages or foam cells (intimal xanthoma) can appear as regions with plaque, since the healthy 3-layered structure of the vessel is not visible. This leads to underestimation of the PFW angle and thus overestimation of the plaque burden. The confounding effect of vessel wall composition may explain why the association between $\mathrm{PFW}_{\mathrm{OCT}}$ and $\mathrm{PB}$ almost vanishes in the more diseased sections. We observe a similar trend in the relation between $\mathrm{PFW}_{\text {IVUS }}$ and $\mathrm{PB}$, although the detection of the PFW by IVUS is usually not affected by attenuation-inducing plaque components. This might indicate a possible biological phenomenon. In the initial stages of plaque growth, the asymmetric plaque growth results in a simultaneously decreasing PFW angle and increasing PB. Apparently in later stages, the plaque growth becomes more symmetrical, decreasing the PFW angle with only a limited increase in PB and thus weakening the relationship. This explanation should be supported by serial imaging studies.

PCI procedures are always guided by angiography to determine catheter position and lumen narrowing. Currently, it is challenging to identify segments seen on OCT with the corresponding location on angiography which could lead to wrong sizing and positioning of the stent. The recently introduced on-line co-registration of OCT and angiography [14] could greatly increase the utility of OCT and thereby the application of our findings in the clinical work flow. Furthermore, the most predictive cut-off angle of $220^{\circ} \mathrm{PFW}$ is hard to determine by eye. This might limit direct clinical application of our findings. New software for the automatic detection of the three wall layers and the determination of the PFW angle is under development in our university.

\section{Limitations}

Some limitations in our study should be taken into account. Despite the use of side branches for optimal matching of OCT and IVUS pullbacks, errors in matching can occur due to intrinsic differences between the two techniques. These include, amongst others, pullback speed, lateral resolution 
and frame rate. To minimize the impact of these mismatches on the accuracy of the results, the measurements were averaged over a region of $3 \mathrm{~mm}$, which is a larger than the maximum expected longitudinal mismatch.

Only a limited number of matched pullbacks were available for analysis. Since we analyzed the IVUS images per cross-section and not longitudinally, we regarded the seven data points per pull-back as separate observations. We do not expect that our conclusion would be much different with a larger dataset. However, these results should still be interpreted as being a proof-of-principle study.

\section{Conclusion}

In conclusion, this study shows that $\mathrm{PFW}_{\mathrm{OCT}}$ is a good predictor for the $\mathrm{PB}$ in the regions with a PFW angle $>186^{\circ}$ and gives more insight in the general interpretation of $\mathrm{PFW}_{\mathrm{OCT}}$ angles. After further clinical validation, this new PB estimation based on OCT could help to identify the optimal landing zone.

Acknowledgments This study was funded by the European Research Council, grant agreement 310457.

\section{Compliance with ethical standards}

The Erasmus MC has a patent licensing agreement with Terumo Corporation. Dr. Van Soest has the rights to receive royalties as part of this agreement. The other authors declare that they have no conflict of interest. Informed consent was obtained from all individual participants included in the study. All procedures performed in this study involving human participants were in accordance with the ethical standards of the institutional research committee and with the $1964 \mathrm{Hel}-$ sinki declaration and its later amendments.

Open Access This article is distributed under the terms of the Creative Commons Attribution 4.0 International License (http://creativecommons.org/licenses/by/4.0/), which permits unrestricted use, distribution, and reproduction in any medium, provided you give appropriate credit to the original author(s) and the source, provide a link to the Creative Commons license, and indicate if changes were made.

\section{References}

1. Tearney GJ, Regar E, Akasaka T et al (2012) Consensus standards for acquisition, measurement, and reporting of intravascular optical coherence tomography studies: a report from the International Working Group for Intravascular Optical Coherence Tomography Standardization and Validation. J Am Coll Cardiol 59:1058-1072. doi:10.1016/j.jacc.2011.09.079

2. Morino Y, Tamiya S, Masuda $\mathrm{N}$ et al (2010) Intravascular ultrasound criteria for determination of optimal longitudinal positioning of sirolimus-eluting stents. Circ J 74:1609-1616

3. Takahashi M, Miyazaki S, Myojo M et al (2015) Impact of the distance from the stent edge to the residual plaque on edge restenosis following everolimus-eluting stent implantation. PLoS One 10:e121079. doi:10.1371/journal.pone.0121079

4. Glagov S, Weisenberg E, Zarins CK et al (1987) Compensatory enlargement of human atherosclerotic coronary arteries. N Engl J Med 22:1371-1375

5. van Soest G, Goderie T, Regar E et al. Atherosclerotic tissue characterization in vivo by optical coherence tomography attenuation imaging. J Biomed Opt 15:011105. doi:10.1117/1.3280271

6. Gogas BD, Garcia-Garcia HM, Onuma Y et al (2013) Edge vascular response after percutaneous coronary intervention: an intracoronary ultrasound and optical coherence tomography appraisal: from radioactive platforms to first- and second-generation drugeluting stents and bioresorbable scaffolds. JACC Cardiovasc Interv 6:211-221. doi:10.1016/j.jcin.2013.01.132

7. Wentzel JJ, Gijsen FJH, van der Giessen R et al (2014) Positive remodeling at 3 year follow up is associated with plaque-free coronary wall segment at baseline: a serial IVUS study. Atherosclerosis 236:82-90. doi:10.1016/j.atherosclerosis.2014.06.013

8. Costa MA, Angiolillo DJ, Tannenbaum M et al (2008) Impact of stent deployment procedural factors on long-term effectiveness and safety of sirolimus-eluting stents (final results of the multicenter prospective STLLR trial). Am J Cardiol 101:1704-1711. doi:10.1016/j.amjcard.2008.02.053

9. Teirstein PS (2010) Drug-eluting stent restenosis: an uncommon yet pervasive problem. Circulation 122:5-7. doi:10.1161/ CIRCULATIONAHA.110.962423

10. Moreno R, Fernández C, Hernández R et al (2005) Drug-eluting stent thrombosis: results from a pooled analysis including 10 randomized studies. J Am Coll Cardiol 45:954-959. doi:10.1016/j. jacc.2004.11.065

11. Hoffmann R, Mintz GS, Mehran R et al (1998) Intravascular ultrasound predictors of angiographic restenosis in lesions treated with Palmaz-Schatz stents. J Am Coll Cardiol 31:43-49

12. Prati F, Romagnoli E, Burzotta F et al (2015) Clinical impact of OCT findings during PCI: the CLI-OPCI II study. JACC Cardiovasc Imaging 8:1297-1305. doi:10.1016/j.jcmg.2015.08.013

13. Kini AS, Motoyama S, Vengrenyuk Y et al (2015) Multimodality intravascular imaging to predict periprocedural myocardial infarction during percutaneous coronary intervention. JACC Cardiovasc Interv 8:937-945. doi:10.1016/j.jcin.2015.03.016

14. Hebsgaard L, Nielsen TM, Tu S et al (2015) Co-registration of optical coherence tomography and X-ray angiography in percutaneous coronary intervention. The does optical coherence tomography optimize revascularization (DOCTOR) fusion study. Int $\mathbf{J}$ Cardiol 182:272-278. doi:10.1016/j.ijcard.2014.12.088 